\title{
Study on the method of judging standard degree of martial arts movement based on computer vision
}

\author{
ZHAO Hong-bo, YAO Wei-hua \\ Institute of Physical Education of Luoyang Normal University, Luoyang, Henan, 471022
}

Keywords: computer vision; motion parameter acquisition; parameter optimization; nonlinear solution

\begin{abstract}
The traditional method of judging standard degree of martial arts movement uses fixed constraint model, it is difficult to describe the dynamic and small area change. The method of judging standard degree of martial arts movement based on computer vision is put forward, computer vision is utilized to capture the movement parameters of martial arts movement, through parameter optimization, the constrained optimization problem of the movement parameters of martial arts movement is regarded as a nonlinear minimized solution problem, L-M motion constraint parameters are utilized to provides a regularization method of fast convergence, so as to obtain movement parameters and structure parameter matrix of martial arts movement, and complete the judgment of standard degree of movements. Simulation results show that the method can accurately judge the standard degree of martial arts movements and improve the measuring precision of parameters.
\end{abstract}

\section{Introduction}

The judgment of the martial arts movement standard level, can improve the skills of athletes, and avoid injury to greatest degree. The judgment of the martial arts movement standard level mainly includes the theory principle of exercise physiology, computer image processing and other fields [1]. In sports, human bones and muscles will produce different behavior information, the comprehensive analysis is carried on movement information of bone and muscle, which can realize the accurate judgment of human body motion parameters [2]. Methods for analyzing martial arts moves standard level used commonly include: the judgment method of the martial arts movement standard level based on main frame image, the judgment method of the martial arts movement standard level based on martial arts action feature modeling, the judgment method of the martial arts movement standard level based on characteristics algorithm of skeletal model [3 4]. Effective analysis of martial arts athlete's motion parameters can improve the motor skills, avoiding occurrence of injuries. Therefore, seek the effective method to judge the standard degree of martial arts moves, maximally avoid martial arts athletes injury problems has become a key issue researched by the related scholars in sports scientific field [5].

\section{The principle of three-dimensional measurement of Martial arts athlete movement's parameters}

The measurement of martial arts athletes movement action parameters is to measure the moving situation of martial arts athletes in two-dimensional space with image sensing device, and transforms it into method of 3D images, and then through the corresponding algorithm to analyze 3D image data, so as to obtain martial arts movement parameters at different spatial coordinates.

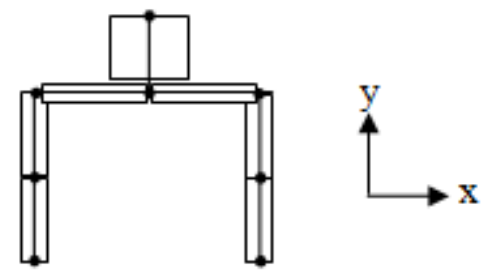

Figure 1 model and coordinate system of human posture 
The traditional methods mostly use motion skeleton model to describe (Figure 1). The coordinate system of construction model can be described by Figure 1 on the right side of figure, the positive direction of coordinate $\mathrm{Z}$ is consistent to the left-handed coordinate system orientation, coordinate origin is at the core position of each joint. The coordinate of a point in three dimensional area is $(w, e)$, and the coordinate of it in the $2 \mathrm{D}$ projection area is $(w, e)$, both of them meet the regulation of equation(1), the change quantity of $\mathrm{Z}$ and $\mathrm{P}$ have linear correlation, through the coordinates of two joints of bone in the image, and invariant bone length constraints, the depth difference of the two joints in three dimensional space can be obtained.

$$
\left(\begin{array}{l}
w \\
e
\end{array}\right)=\frac{1}{p}\left(\begin{array}{lll}
1 & 0 & 0 \\
0 & 1 & 0
\end{array}\right)\left(\begin{array}{l}
x \\
y \\
z
\end{array}\right)
$$

\section{Method of judging standard degree of martial arts movement based on computer vision}

The algorithm analysis.The prediction process of martial arts movement parameters, can be considered as the process to obtain the minimum movement parameters, the motion function is described with $y=g(x)$, where $p$ is the $p$ dimension vector, $q$ is a $q$ dimension vector. Generally, $y=g(x)$ can be viewed as the measurement vector of actual value $y=g(x)$, specific expression as shown in formula (2):

$$
\min \|Y-g(X)\|
$$

The L-M method is an optimized first-order Newton iterative algorithm, which can be used to treat the optimization of mass parameters timely and effectively. The process of searching the optimal point with L-M is illustrated in figure 2. The specific operation process of first order Newton iterative method:

Assuming that the original solution is $x^{h}$, make $g^{h}=Y-g\left(x^{h}\right)$. If the value around the $x^{h}$ of $g$ can be obtained by $g\left(x^{h}+\Delta^{h}\right)=g\left(x^{h}\right)+H_{x} \Delta^{h}$ (first order) approximately, where $H_{x^{h}}=\frac{\partial x}{\partial y} \mid x=x^{h}$ represents the value of the Jacobean matrix of $g$ at $x^{h}$. The next iterative point $x^{h+1}=x^{h}+\Delta^{h}$ have to be acquired to ensure that the formula(3) is set up :

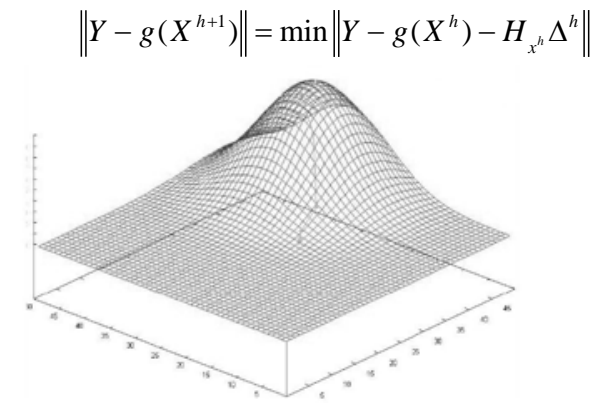

Figure 2 the image description of L-M iterative algorithm process

The minimized standardization equations eventually obtained as:

$$
G^{h} \Delta^{h}=H^{R} l^{h}\left(G^{h}=H^{R}{ }_{X^{h}} H_{X^{h}}\right)
$$

Therefore, one order Newton iterative points sequence is:

$$
X^{h+1}=X^{h}+H_{X^{h}}\left(Y-g\left(X^{h}\right)\right)
$$

The process of two order Newton is same to first order Newton method, the speed of convergence has a strong correlation with the original value $\bar{G} \Delta=H_{X}$. The Liebenberg-Marquardt method utilize incremental standard equation:

$$
\bar{G} \Delta=H_{X}
$$

Standard equation in the Newton method is updated, wherein, $\bar{G}_{i j}=(1+\mu) G_{i j}$, if $\bar{G}_{i j}=G_{i j}$, then $\bar{G}_{i j}=G_{i j}$. The ultimate goal of the L-M method iterative procedure is to search the optimal value of $\mu$. The operation process of L-M algorithm is: 
1) select original point $\eta$, stop the control constant $\eta$, and operate $l^{0}=\left\|Y-g\left(x^{0}\right)\right\|$ to $\mathrm{r}=0, \mu_{0}=10^{-3}$.

2) Operate $H_{x^{h}}=\frac{\partial x}{\partial y} \mid x=x^{h}$, and establish the increment standard equation: $\bar{G}\left(\mu^{R}\right) \Delta^{R}=H_{x^{h} c^{h}}$.

3) solve the increment standard equation to obtain $\mathrm{r} \Delta^{R}$. If $\left\|Y-g\left(x^{h}+\Delta^{h}\right)\right\| \geq l^{h}$, then $x^{h+1}=x^{R}+\Delta^{R}$; if $\left\|\Delta^{R}\right\|<\kappa$, then terminate the iteration, and output the results; otherwise, let $\mu^{h+1}=10 \mu^{h}, \quad \mu^{h+1}=10 \mu^{h}$, and go to step 4.

4) If $\mu^{h+1}=10 \mu^{h}$, let $\mu^{h+1}=10 \mu^{h}$ and solve the standard equation $\Delta^{h}$ again.

Estimation of martial arts movement action parameter. Assuming the matrix of measured martial arts moves action feature point is $\left[\begin{array}{c}w_{i j} \\ e_{i j}\end{array}\right], i=1, \ldots G, j=1, \ldots Q$, the matrix of image feature points which need to be predicted is $\left[\begin{array}{c}w_{i j} \\ e_{i j}\end{array}\right], i=1, \ldots G, j=1, \ldots Q$. If the measurement matrix constituted of characteristic point of each frame image $\left[\begin{array}{c}w_{i j} \\ e_{i j}\end{array}\right], i=1, \ldots G, j=1, \ldots Q$ is $G$, wherein $G$ represents the image frames, $Q$ is the number of characteristic points of the single frame images, the ultimate goal of the matrix is to obtain the three-dimensional structure $\tilde{Z}_{i 3 \times Q}$ of each frame moving image and rotation matrix $W_{i 3 \times 3}$. If the 3D shape of athletes' joint is weighted linear set of shape base, there are: $\tilde{Z}_{i}=\sum_{i=1}^{R} \gamma_{i j} Z_{i}$. Among them, $\gamma_{i j}$ is the weighted coefficient, $Z_{i}$ represents the shape base, $R$ denotes the number of shape base. If $R=1, \gamma_{i j}$, then the state of key area under the weak perspective projection model is:

$$
x_{i j}=\left[\begin{array}{c}
w_{i 1, \ldots, \ldots}, w_{i Q} \\
e_{i 1, \ldots,}, e_{i Q}
\end{array}\right]=\bar{W}\left(\sum_{i=1}^{R} \gamma_{i j} Z_{i}\right)+\bar{D}_{i} g_{m}^{D}
$$

In the formula, $\bar{W}_{i}$ represents the first two rows of the rotation matrix $W_{i 3 \times 3}, \bar{D}_{i}$ represents the first two elements of the translation vector $D_{i 3 \times 1}$, and $g_{m}^{D}=[1, \ldots, 1]_{1 \times m}$. In order to optimize operation process of algorithm, and then filter the translation vector $\left\{\begin{array}{l}\bar{w}_{i j}=w_{i j}-\frac{1}{Q} \sum_{j=1}^{Q} w_{i j} \\ \bar{e}_{i j}=e_{i j}-\frac{1}{Q} \sum_{j=1}^{Q} e_{i j}\end{array}\right.$, processes are shown by formula (8):

$$
\left\{\begin{array}{l}
\bar{w}_{i j}=w_{i j}-\frac{1}{Q} \sum_{j=1}^{Q} w_{i j} \\
\bar{e}_{i j}=e_{i j}-\frac{1}{Q} \sum_{j=1}^{Q} e_{i j}
\end{array}\right.
$$

The formula (8) is further processed to get:

$$
x_{i j}=\left[\begin{array}{l}
\bar{w}_{i 1, ., \ldots}, \bar{w}_{i Q} \\
\bar{e}_{i 1, \ldots, .,} \bar{e}_{i Q}
\end{array}\right]=\bar{W}\left(\sum_{i=1}^{R} \gamma_{i j} Z_{i}\right)
$$

Ensure that the projection error minimization of $w$ and $w$, so as to obtain the motion parameters matrix $W$ and 3D structure parameters matrix $\gamma_{i, h} P_{h}$, this process is shown in formula (10):

$$
\min \sum_{i, j}\left\|x_{i j}-x_{i j}^{\prime}\right\|^{2}=\min \sum_{i, j}\left\|x_{i j}-\left(W_{H} \sum_{h} \gamma_{i, h} P_{h}\right)\right\|^{2}
$$

The method through quaternion approach to express the action parameter matrix $w_{g}$, but this method needs Gauss-Newton iterative analysis repeatedly, so, computation of algorithm is increasing when parameters is large, which makes the accuracy of the algorithm decrease. This paper can solve the shortcomings by the sparse feature of Jacobi matrices. 


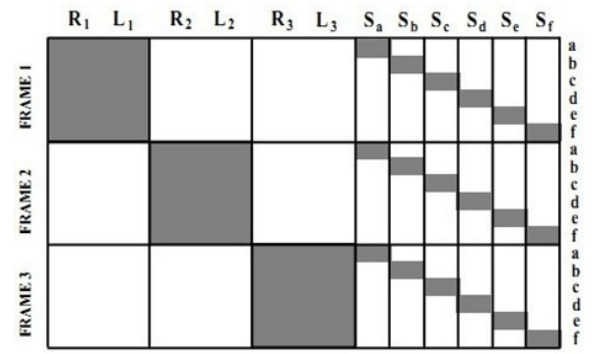

Figure 3 The sparse characteristics of Jacobi matrix of six feature points in three images

The movement structure can be quantified into $3 R \times Q$ coordinates and $R \times G$ deformation coefficient $\gamma_{\text {in }}$ about $P_{R}$ shape base. With the improved method to analyze the key target in motion structure parameters and obtain the reasonable original value.

The initialization process of shape base coefficient $\gamma_{i h}$ is analyzed based on the rigid features, the nonlinear optimization method will have local optimal phenomenon in the iteration, cause all 3D points tent to the same plane. Therefore, an original 3D shape should be integrated into the improved objective function, the specific process as shown in formula (11):

$$
\sum_{i=2, j=1}^{i=G, j=Q}\left\|P_{L}^{i-1}-P_{L}^{i, j}\right\|^{2}
$$

The method can solve the problem of unable to effectively adjust the constraint information, when time interval of image sequence is small.

\section{Experimental verification}

Through the computer simulation to demonstrate the validity of this algorithm. The time interval of experimental sample acquisition is $0.5 \mathrm{~s}$, feature points of each frame image acquisition is 500 . Comprehensive analysis is processed for movement characteristics of martial arts athletes during the movement experiment, the model effect of two kinds of action is obtained as shown in figure 4:

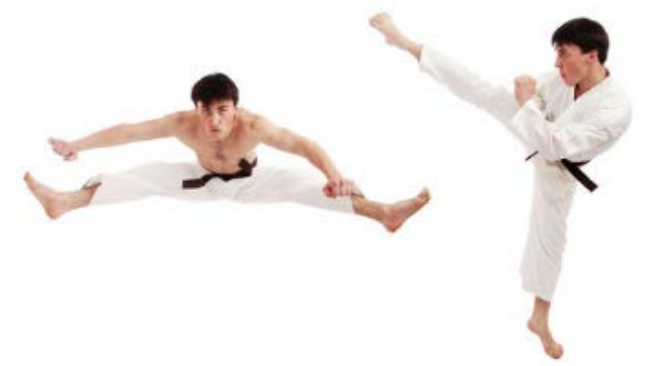

Figure 4 the sample image in database

Using the traditional algorithm to analyze the standard level of martial arts movement, the analytical results obtained as shown in figure 5:

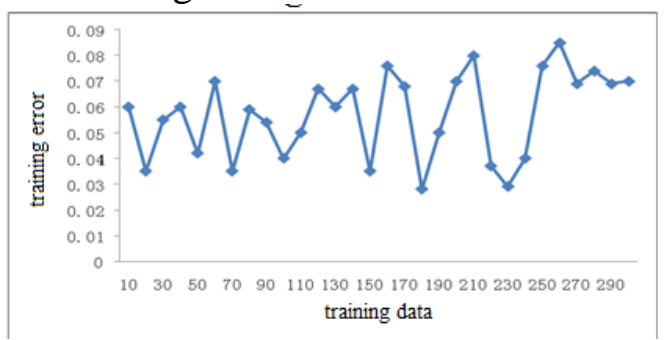

Fig. 5 the judgment results of the traditional algorithm

Using the proposed algorithm to analyze human motion behavior, the analytical results obtained as shown in figure 6: 


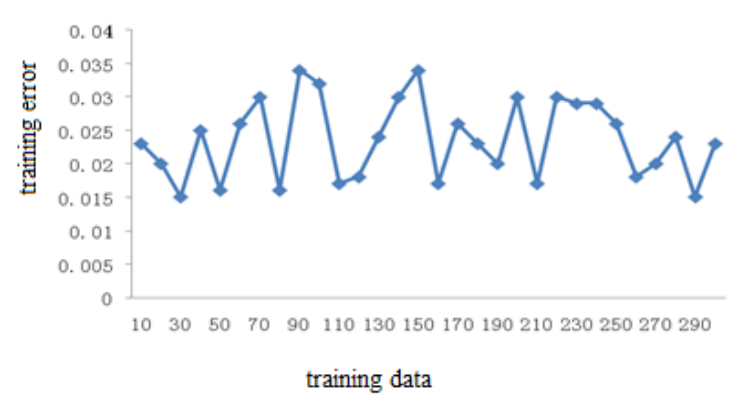

Figure 6 the judgment results of the proposed algorithm

By analyzing Figure 5 and Figure 6, it can be learnt that the feature of forecasting data obtained by the proposed method and data in database have high matching degree. In order to further verify the superiority of the algorithm, 6 motion parameters are chosen randomly in the sample database, respectively, the proposed algorithm and traditional algorithm are adopted to measure it respectively. Among them, $\theta_{1}$ and $\theta_{2}$ represent deflection angle of skeleton of two selected sites respectively, $s_{1}$ and $s_{2}$ represent degree of shrinkage of two selected muscles respectively. The obtained results shown in table 1 :

Table 1 the sample data in database

\begin{tabular}{lllll}
\hline Behavior sample number & $\theta_{1}$ & $\theta_{2}$ & $s_{1}$ & $s_{2}$ \\
\hline 1 & 109 & 56 & 76 & 57 \\
2 & 68 & 48 & 36 & 48 \\
3 & 34 & 22 & 48 & 56 \\
4 & 20 & 28 & 60 & 22 \\
5 & 79 & 34 & 95 & 4 \\
6 & 103 & 56 & 67 & 48 \\
\hline
\end{tabular}

Using the traditional algorithm to analyze posture parameters, obtained body posture parameters are shown in table 2:

Table 2 the judgment data of the traditional algorithm

\begin{tabular}{lllll}
\hline Behavior sample number & $\theta_{1}$ & $\theta_{2}$ & $s_{1}$ & $s_{2}$ \\
\hline 1 & 102 & 52 & 71 & 53 \\
2 & 64 & 53 & 32 & 42 \\
3 & 21 & 7 & 43 & 59 \\
4 & 27 & 34 & 65 & 5 \\
5 & 86 & 39 & 92 & 23 \\
6 & 99 & 52 & 72 & 53 \\
\hline
\end{tabular}

Using the proposed algorithm to analyze human behavior, obtained human posture data are shown in table 3:

Table 3 the judgment data of the proposed algorithm

\begin{tabular}{lllll}
\hline Behavior sample number & $\theta_{1}$ & $\theta_{2}$ & $s_{1}$ & $s_{2}$ \\
\hline 1 & 108 & 57 & 76 & 58 \\
2 & 69 & 48 & 37 & 42 \\
3 & 34 & 24 & 48 & 56 \\
4 & 22 & 26 & 62 & 24 \\
5 & 79 & 35 & 97 & 5 \\
6 & 104 & 56 & 68 & 48
\end{tabular}

By analyzing the data in Table 1, table 2 and table 3 data, it can be seen that using the proposed algorithm to analyze human behavior, the matching of the results of analysis with the database 
samples is higher, it shows that the proposed algorithm have higher accuracy of judging martial arts moves standard degree than the traditional algorithm, and achieved satisfactory effect.

\section{Conclusions}

The method of judging standard degree of martial arts movement based on computer vision is put forward, computer vision is utilized to capture the movement parameters of martial arts movement, through parameter optimization, the constrained optimization problem of the movement parameters of martial arts movement is regarded as a nonlinear minimized solution problem, L-M motion constraint parameters are utilized to provides a regularization method of fast convergence, so as to obtain movement parameters and structure parameter matrix of martial arts movement, and complete the judgment of standard degree of movements. Simulation results show that the method can accurately judge the standard degree of martial arts movements and improve the measuring precision of parameters.

\section{References}

[1] Han Xiaoning. Simulation Research on the motion of virtual human [D]. Xi'an Electronic and Science University. Master's degree thesis.2006.3.34-38

[2] Ren Lifeng. Research of virtual human technology in virtual environment [D]. Zhejiang University. Doctoral dissertation.2008.6.60-64

[3] Magnenat-Thalmann N, Laperriere R, Thalmann D. Joint-Dependent Local Deformations for hand animation and object grasping[C]. Proc. 'Graphics Interface 88, Edmonton. 1988

[4] L Wang, W Hu and $\mathrm{T}$ Tan. Recent Developments in Human Motion Analysis. Pattern Recognition, 2003, 36 (3): 585-601.

[5] Huang Haiming. Study on motion control technology of capturing virtual human movement [D]. Chinese Academy of Sciences. Doctoral dissertation.2007.4.29-93

[6] Zhang Fan, Cao Xibin, Zou Jingxiang. A new conversion algorithm based on all angle quaternion and Euler angles [J]. Journal of Nanjing University of Science and Technology (NATURAL SCIENCE EDITION).2002 (04): 376 - 380

[7] Liu Kai, Chai Yi, Feng Wenwu. Simulation of Human posture data based on OPENGL [J]. Computer simulation.2009 (04): 267-270.

[8] Dai Xiaoqing. Research on motion capture technology based on motion retargeting [D]. Tianjin University. Master's degree thesis.2007.8.67-70 